slight improvement. The albumin diminished in quantity, the headaches and giddiness were less severe, but the cedema increased until it extended to the vulva and the loins. On the 28th I advised copious daily enemata of soap-andwater and discontinued the jalap. Labour commenced on Way 1st and the first stage lasted about 24 hours, the vertex tresenting. About noon on the 2nd I ruptured the membranes and the head quickly descended on to the perineum, when the patient complained of great giddiness and shortly atterwards without any further warning became convulsed, the fit lasting several minutes. The child was born almost immediately afterwards and the placenta was expressed without difficulty. I gave her a draught containing liquid extract of ergot with 20 grains each of chloral hydrate and bromide of potassium. Half an hour after the birth of the child she had another attack of convulsions lasting for four or five minutes and followed by a short period of semiconsciousness and great exhaustion, the pulse going up to 140. When I left the house an hour later she had quite recovered consciousness and speech and had had no return of the convulsions, from which she did not subsequently suffer. A week af terwards the urine still contained a trace of albumin (about one-twelfth), and by the end of a fortnight it was normal. The odema gradually disappeared concurrently with the disappearance of the albumin and the patient made an uneventful recovery, though she remained anæmic for some weeks.

With the exception of rupture of the uterus and concealed accidental hæmorrhage there is probably no more fatal complication met with in the practice of midwifery than eclampsia. Its serious nature, as well as its comparative rarity and its obscure pathology, have combined to invest this disease with such interest and importance that every case seems worthy of record. Many theories have been advanced from time to time as to the cause of the convulsions, but the etiology of the disease is still much disputed. There is general agreement amongst pathologists and obstetricians that it is due to a toxæmia, but while according to some authorities the origin of the toxin is to be found in some pathological condition of the placenta or the fotus giving rise to bio-chemical changes, others deny the influence of either placental or foetal conditions and maintain that it always arises from some morbid maternal state. Probably the most generally accepted view is that the disease "is due to a toxæmia of a urinary nature, toxic substances being retained in the blood which should have been excreted by the kidneys," 1 and the frequent association of albuminuria with eclampsia certainly supports this theory. It has been stated ${ }^{2}$ that one pregnant woman in 40 is albuminuric, and that of these one out of every four develops eclampsia; that eclampsia withont albuminuria is of rare occurrence-one case in 10 cases. From these facts the practical conclusion is drawn that albuminuria should always be looked for in pregnant women, and actively treated during pregnancy when detected.

As regards treatment there can be no doubt that the chief indication is to eliminate the toxic matter from the circulation, or, failing this, to dilute it as much as possible. With this object in view purgatives, diuretics, and diaphoretics are given, and Dr. R. Jardine has recently strongly advocated the use of saline injections. In the two first cases above recorded I relied on calomel and croton oil respectively as purgatives, in each instance with very satisfactory results; but in any future case I should feel more inclined to resort to the more rapid and certain method of emptying the lower bowel with copious and repeated enemata. The second indication must be to counteract or modify the effects of the poison in the system, and for this purpose numerous drugs have been recommended-morphia, chloral hydrate, bromides, chloroform, veratrum viride, \&c. After the experience gained in the three cases now described I should never hesitate to place entire reliance on the mixture of chloral hydrate and bromide, supplemented if need be by the prolonged administration of chloroform; but in a disease of such gravity, arising from a toxin, whatever its nature, of such virulence, it is absolutely necessary to give these counteracting drugs in full doses, and above all to persevere in the line of treatment adopted and that even in cases apparently hopeless.

Commercial-road East, E.

1 Dr. R. Jardine : Thr LANCET, July 1st, 1899, p. 28.

2 Charles : Journal d'Accouchements, April 3rd, 1898.

\section{A FEW CASES OF BRAIN INJURY CLINICALLY EXAMINED.}

BY EDMLND E. DYER, M.B. EDIN., SURGEON TO CLACR MANNAN COUNTY HOSPITAT.

As the clinical study of head affections and injuries, when verified by post-mortem examination, is always interesting, the following cases may be of more than ordinary interest, more particularly as each of the following cases shows the enormous amount of brain tissue which may be destroyed without causing at least immediate death.

CASE 1.-The first case was that of a man, aged 20 years, who was admitted into the Clackmannan County Hos. pital suffering from a compound fracture of the skull which he sustained by being knocked down by the revolving handle of a steam winch. On admission his breathing was laboured and stertorous, there was considerable muscular twitching, and just after the accident he had had several violent convulsions. There was considerable hæmorrbage from the wound, which was about three inches above the left ear. He was insensible except to strong stimuli. There was a considerable scalp wound, with extreme shattering of bone which was also much depressed. I decided to trephine at once and having had the patient put under chloroform I removed entirely a triangular piece of bone measuring two inches by one and three-quarter inches by one and a quarter inches, and also two other pieces of small bone which had been crushed down from the inner table. The dura mater was to the naked eye apparently uninjured. As there was still a great deal of depression $I$ inserted my finger between the dura mater and the frontal bone and lifted up the whole, when it went back into place with a snap. Immediately afterwards the breathing became regular and quite natural. The external wound, which was originally two and three-quarter inches in length, running upwards and backwards, and which was extended for operation to five inches, was closed, and a drainage tube was put in, which was taken out on the third day, the wound having practically healed without suppuration. The patient gradually recovered consciousness and on about the fifth day he was able to ask for anything. Curiously enough, from the day after the injury he insisted on getting out of bed for the purpose of passing urine, and this in spite of all attempts to prevent him. On the ninth day a reddish swelling and general puffiness developed over the nose and forehead which at first looked almost erysipelatous in character, and on the next day the temperature rose to $1024^{\circ} \mathrm{F}$. This, however, went down on the fourteenth day, when he gradually improved and spoke a little, and could answer questions if time was given to him, although cerebration was very slow. The improvement continued and on the seventeenth day he sat up in bed and ate a small piece of meat and complained that it was not enough. On the twenty-first day a swelling similar to that which developed on the forehead came on the posterior occipital parietal region. On the twenty-fourth day the patient developed extreme photophobia. He lay on his left side and curled himself up. He became comatose on the twenty-sixth day and died two days after, or 28 days after the injury. As the case fell under the scope of the Fatal Accidents Inquiry Act (Scotland), 1895, I made a post-mortem examination with a brother practitioner and we found the following conditions. The external wound was quite healed and the dura mater had thickened slightly at the point of injury. On reflecting the scalp the fracture was found to extend down to the left ear, right down to the left orbital plate, then right across the head posterior to the fronto-parietal suture to near the right ear. On lifting the skull-cap the dura mater was found to be adherent to it at the seat of injury. Below the dura mater the brain was found to be more or less covered with pus and immediately below the seat of injury was an abscess. The brain was removed and on making sections the abscess was found to extend from the point of injury, which was on the middle frontal convolution backwards and inwards, to the lateral ventricle. In the right cerebral hemisphere another abscess was found just below the surface of the brain at the angular convolution extending to a point close to the lateral ventricle 
on that side. Pus was also found over the base of the brain and around the spinal cord.

In reviewing this case I can only suppose that the cedema over the frontal region was due to the abscess on the left side and that it went down with the contemporaneous fall of temperature when the tension was relieved by the pus bursting into the lateral ventricle. The second abscess did not find such exit. The other points to consider clinically in the case are the absence of paralysis, the extraordinary destruction of brain tissue, the extreme photophobia, and, finally, the length of time under the circumstances that the patient lived after the injury.

CASE 2.- The patient was an old soldier, aged 56 years, whom I admitted into the hospital suffering from right hemiplegia. The man had been ill for several days before his admission. No very definite history could be got. He was able to speak a few words, but cerebration was very slow, and on the left eye there were signs of an old keratitis. On the eighth day after his admission he became worse generally and he suddenly developed most violent convulsions which lasted for several hours, but which gradually subsided after treatment with heroic doses of chloral and morphia. From this date the convulsions never recurred, but there were slight twitchings from time to time. The patient died on the twenty-fourth day after his admission into the hospital and about the thirtieth day after the first seizure. Death occurred in the way so common with many of these cases except that the man took his nourishment well up to the day of his death. The temperature never rose above $99 \cdot 8^{\circ} \mathrm{F}$. There was no affection of the breathing.

The interest of this case lies chiefly in the postmortem examination which, happily, I was able to make. On opening the head the dura mater was found to be adherent to the bone in patches all over the calvarium and was detached only with great difficulty in some places. The veins of the dura mater were enormously dilated. The whole brain tissue was extremely soft. On making sections of the brain horizontally the first thing which was discovered was a large round clot flattened from above downwards, measuring one and three-quarter inches in diameter, placed high up just inside the fissure of Rolando. On making further sections a well-organised smaller clot was found a little lower down just inside the middle frontal convolution, and again at a lower level a clot, of much the same size and character as the last, situate in the occipital region. These three clots were all found on the left side of the brain, but on making similar sections on the right side three other clots were found in almost exactly corresponding positions. The only difference was that the oldest clot of all-viz, the one in the frontal region-was here and the most recent clot of all was on this side in the occipital region. The last clot found was on the left side of the cerebellum and was three-quarters of an inch in diameter.

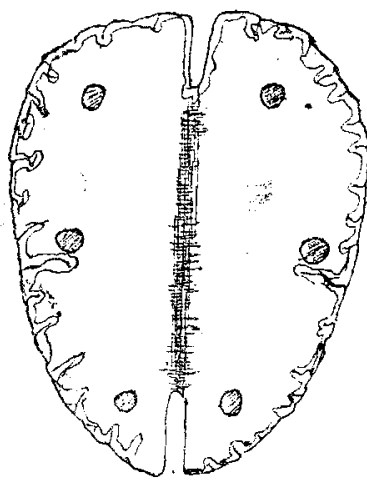

Diagram representing the relative position of clots in the brain in Case 2. Six clots are shown. The seventh clot was in the body of the cerebellum. The clots were not upon the same level, although diagrammatically they are so
represented.

Now, which clot or clots, if any, caused the convulsions? From the date of the first symptoms and comparing the oldest clot with the more recent ones and considering the time which elapsed between the convulsions and the date of death I am inclined on these grounds alone to think it was the clot in the cerebellum. Although the age of the clot below the fissure of Rolando was much the same as that in the cerebellum, yet, on the other hand, the age of the corresponding clot below the fissure on the other side was distinctly different, and had this been the cause then it is reasonable to infer that instead of one set of general convulsions there woold have been another at a later period, and this was not the case. The chief point of interest about this case is the number of separate hæmorrhages, no less than seven, each one beins of a different age, and as far as affected the cerebrum almost exactly symmetrical ; further, that so far as could be seen only one of these hæmorrhages caused the convulsions. The internal capsule was not affected and in no case apparently did the clot affect the grey matter of the brain. The man was an alcoholic and from the signs which were found I have little doubt that he had suffered from syphilis. In this case also there was no affection of the breathing.

CASE 3.-The third case is perbaps as interesting as the foregoing in showing an extensive destruction of brain tissue without a fatal ending. A lad was admitted into the hospital suffering from an injury to his head. It had been crushed from side to side between a fixed upright beam and the arm of a machine working horizontally, the point of which was as nearly as possible three-quarters of an inch square. On admission he was bleeding freely from a small cut over the right temple and a quarter of an inch behind the temporal artery. The injury to the bone was slightly to one side of the cut and to the front. I introduced a probe very gently and found a small fracture at the point indicated and with so much destruction of brain-tissue that the probe by its own weight entered exactly two and a half inches straight into the brain. Considering the condition of the patient and the absence of any urgent symptoms I did not think it a case for operation. The bleeding was stopped, an antiseptic pad put on, and the boy was sent to bed. The external wound, which was not sewn, healed without trouble and the patient was discharged in about three weeks. The boy after receiving the injury walked about 200 yards from the works to a medical man. But for a little faintness and pain for a few days the patient suffered no inconvenience. There was no rise of temperature or bad symptiom.

Each of these cases shows the enormous amount of brain. tissue which may be destroyed and, further, the extraordinary efforts the brain is capable of exerting to repair the damage, both effort and power being quite beyond what is often supposed it is capable of exerting.

Alloa, N.B.

\section{SURGICAL ASPECTS OF CONSTIPATION.}

\section{BY S. L. WOOLMER, M.R.C.S. ENG.}

The following two cases are recorded in order to demon. strate what is believed to be a rare sequel to the most comanon morbid condition known to exist. I can find in no works on modern surgery any reference to a case of "aneurysmal" dilatation of the colon as the result of chronic constipation, and yet that it must exist in no small degree is very obvious. Due to this dilatation and the consequent accumulation of fæcal matter therein the condition of both patients was reduced to one of great gravity, as witnessed by the repeated attacks of intestinal obstruction, the degree of anæmia produced by insufficient alimentation, and stercoræmia. No enema could have possibly cleared the intestine nor could any procedure short of operation have relieved the patients.

CASE 1.-A man, aged 28 years, was under the care of Dr. Masi in the Military Hospital of Buenos Ayres. $\mathrm{He}$ had been constipated for four years. On Dec. 23rd, 1892, during a long march, and after a meal which consisted principally of cheese, he could obtain no move. ment of his bowels, and in this state he continued till March 12th, 1893, having only been able to expel by the rectum gases and a little blood. He then entered the Hospital San Roque in Buenos Ayres, where he was eventually relieved. From this period till November, 1896, he continued always constipated, relieving himself only by enemas and purges, and then with great difficulty. In November, 1896, he entered the military hospital under the care of Dr. Masi, where he recovered somewhat his functions by the application of abdominal massage and enemas of half a litre of olive oil administered daily. The presence now of blood in the motions and their ribbon-like character persuaded Dr. Masi that he had to deal with a malignant disease of the colon and he considered, therefore, the advisability of performing laparotomy. 